\title{
Efecto del consumo de chía (Salvia hispánica) sobre los síntomas de estreñimiento que presentan los estudiantes de una universidad particular de Lima Este, 2014
}

\author{
Effect of consumption of chia (Salvia hispanica) on constipation symptoms presented by students \\ from a private university in Lima Este, 2014
}

Eidi Bernal Altamirano ${ }^{1}$, Johanna Jessenia Iñaguazo Trávez², Bertha Chanducas Lozano ${ }^{3}$

\begin{abstract}
RESUMEN
Objetivo: Este trabajo pretende determinar el efecto del consumo de chía (Salvia hispánica) sobre los síntomas de estreñimiento que presentan los estudiantes de una universidad privada de Lima Este. Material y Métodos: El estudio es de diseño pre-experimental y de corte longitudinal. Los participantes fueron 20 estudiantes entre 18 a 25 años con síntomas de estreñimiento. Para el tratamiento fueron divididos en dos subgrupos, donde el subgrupo A consumió 25 gr. y el subgrupo B 15 gr. de semillas de chía gelatinificada. Resultados: Según el diagrama de tendencia que se realizó, al inicio del tratamiento todos presentaron los síntomas de estreñimiento. Luego, en la primera y segunda semana del tratamiento ya se observa una disminución considerable de los síntomas de estreñimiento, y en la tercera semana solo dos personas de los subgrupos A y B presentaban los síntomas de estreñimiento. Conclusiones: En síntesis, se encontró que existe diferencia significativa entre el pre y pos test con respecto a los síntomas de estreñimiento de los participantes de este estudio, siendo corroborado con la prueba de McNemar $(\mathrm{p}<0.05)$.
\end{abstract}

Palabras clave: Estreñimiento, Consumo de las semillas de chia, Fibra dietaria

\begin{abstract}
Objetive: This research pretends to determine the effect of consumption of chia seed (Hispanic sage) about the symptoms of constipation which presents the students of a private university of east Lima, 2014. Methods: The study is pre-experimental design and longitudinal cut. Results: The Treatment was applied in the group of students with constipation for 3 weeks, which were divided into two subgroups consuming gelatin chia seeds 25gr the Subgroup A and $15 \mathrm{gr}$ the subgroup B. Conclusions: It was concluded that there is a significant decrease in symptoms of the constipation symptoms in the third week of treatment in both groups, being corroborated with the McNemar test $(\mathrm{p}<0.05)$.
\end{abstract}

Keywords: Constipation, consumption of Chia seed, dietary fiber.

\footnotetext{
${ }^{1}$ Nutricionista, Centro de Salud de Puerto Ocopa, Distrito del Río Tambo, Zatipo, Junín, Perú.

${ }^{2}$ Nutricionista, Colegio Adventista Emanuel, Lago Agrio, Quito, Ecuador.

${ }^{3}$ Docente EP Nutrición Humana, Facultad de Ciencias de la Salud, Universidad Peruana Unión, Lima Perú.
} 
Efecto del consumo de chía (Salvia hispánica) sobre los síntomas de estreñimiento que presentan los estudiantes de una universidad particular de Lima Este, 2014

\section{INTRODUCCIÓN}

Rivera (2006) menciona que, en los últimos años, en los estudiantes universitarios la frecuencia de consumo de alimentos refinados pobres en fibra, ricos en grasas y azucares coincide más con la alimentación de países industrializados, el cual es un factor causal de numerosas enfermedades como es el estreñimiento crónico (Botella et al., 2011). Este problema se puede mejorar y prevenir con una dosis adecuada de fibra (Rubio, 2002). Actualmente las semillas de chía son fuentes ricas en fibra dietética (Salgado, 2005; Garda, 2012) por su equilibrada relación de fibra soluble e insoluble (Fuentes, 2012), las cuales pueden ser consumidas de manera directa o trasformadas en productos ricos en fibra (Gonzales, 2010). Lujan (2011) indica que la importancia de las semillas de chía radica en la capacidad de absorber grandes cantidades de agua (15.41g. de agua/g de fibra) comparado con otra fuente de fibra como el salvado de trigo (2.48g de agua/g. de fibra), el cual tiene efectos fisiológicos al aumentar el bolón fecal y acelerar el tránsito intestinal.

Higgins (2004) revela que uno de los problemas más comunes del aparato digestivo es el estreñimiento con una prevalencia en la población mundial del $27 \%$, de los cuales solo el 5\% acude a consulta médica. En el Perú, el MINSA (2004) presentó estadísticas del estreñimiento donde señala que es un problema que afecta más a las mujeres de 25 a 55 años, esto se debe a que su composición hormonal es diferente al del hombre. Remes et al. (2009) refieren que el estreñimiento afecta a los jóvenes y a los ancianos con frecuencia similares.

La fibra dietética es un conjunto de sustancias de origen vegetal que resisten a la digestión y absorción en el intestino delgado humano y experimenta una fermentación total o parcial en el intestino grueso (Royo, 2007). Es un elemento importante para una nutrición sana, desempeñando funciones fisiológicas sumamente importantes como estimular el tránsito intestinal. Solamente se encuentra en alimentos de origen vegetal como la semilla de chía (Tomas, 2013).

La fibra soluble está formado por distintos componentes como mucilagos, inulina, pectinas, gomas y fructooligosacáridos, que captan una gran cantidad de agua y son capaces de formar geles viscosos, siendo fermentable por microorganismos presentes en el intestino, por lo que produce gran cantidad de gas, al ser muy fermentable favorece la creación de la flora bacteriana que compone $1 / 3$ volumen fecal, por lo que este tipo de fibra también aumenta el volumen de las heces y disminuye su consistencia, la podemos encontrar en la semilla de chía (Lujan, 2011). El mucílago de las semillas de chía (Figura 1) es un polisacárido de alto peso molecular, se encuentra en las tres capas exteriores de la cubierta de la semilla. Cuando la semilla entra en contacto con el agua, el mucílago emerge inmediatamente y en un corto periodo se forma un "cápsula mucilaginosa" transparente que rodea la semilla (Guiotto, 2014).

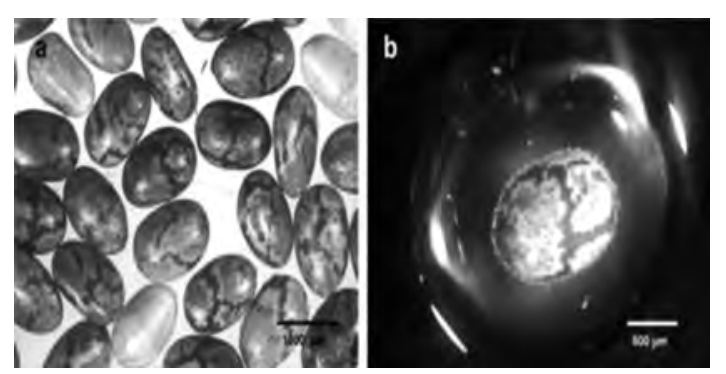

Figura 1

Micrografías ópticas de semillas de chía (a) semillas secas y enteras (b) semillas entera hidratada con la formación de la "Capsula mucilaginosa.

\section{MATERIAL Y MÉTODOS}

Se trata de una investigación de diseño preexperimental y de corte longitudinal

(García, 2012).

El esquema del diseño pre experimental se diagramaría así:

\begin{tabular}{|llll|}
\hline $\mathrm{G} \mathrm{O}$ & $\mathrm{O}_{1}$ & $\mathrm{X}$ & $\mathrm{O}_{2}$ \\
\hline
\end{tabular}

\section{Donde:}

G: Grupo de estudiantes con síntomas de estreñimiento. $\mathrm{O}_{1}$ : Nivel de los síntomas de estreñimiento en el pre test. $\mathrm{O}_{2}$ : Nivel de los síntomas de estreñimiento en el post test. X: Aplicación del tratamiento (Consumo de Chía).

Los participantes fueron 20 estudiantes, se obtuvo mediante el muestreo no probabilístico por bola de nieve o llamada también en cadena (García, 2012). Los sujetos de estudio fueron estudiantes con síntomas de estreñimiento de las facultades de Teología y Ciencias de la Salud de la Universidad Peruana Unión con edades entre 18 a 25 años (Tabla 1)

La información se obtuvo mediante la aplicación de una encuesta que tenía 5 partes: Información sociodemográficos, Criterios de Roma III para 
diagnosticar el estreñimiento crónico, recordatorio de 24 horas un método que proporciona una estimación cuantitativa y cualitativa de la ingesta de un alimento, frecuencia de consumo de alimentos que permite ver el consumo habitual a largo plazo de los alimentos y la hoja de seguimiento semanal que ayudo a tener un control y seguimiento sobre el consumo de chía y la disminución de los síntomas de estreñimiento.

El tratamiento consistía en consumir semillas de chía en 200 cc de agua previo remojo de 15 a 30 minutos durante tres semanas (21 días). Para el tratamiento los participantes fueron divididos en dos subgrupos (A y B), donde el subgrupo A consumió 25 gr y el subgrupo B consumió 15 gr de semillas de chía. Para demostrar la efectividad del consumo de chía en la disminución de los síntomas de estreñimiento se utilizó la prueba de McNemar. Esta prueba analiza variables de tipo dicotómico, tales como: positivo y negativo, hembra y macho, presencia y ausencia donde se denomina “0” y “ 1 ”. Además esta prueba se utiliza para comparar la muestra antes y después de un tratamiento (Berenson \& Levine, 1996).

\section{RESULTADOS}

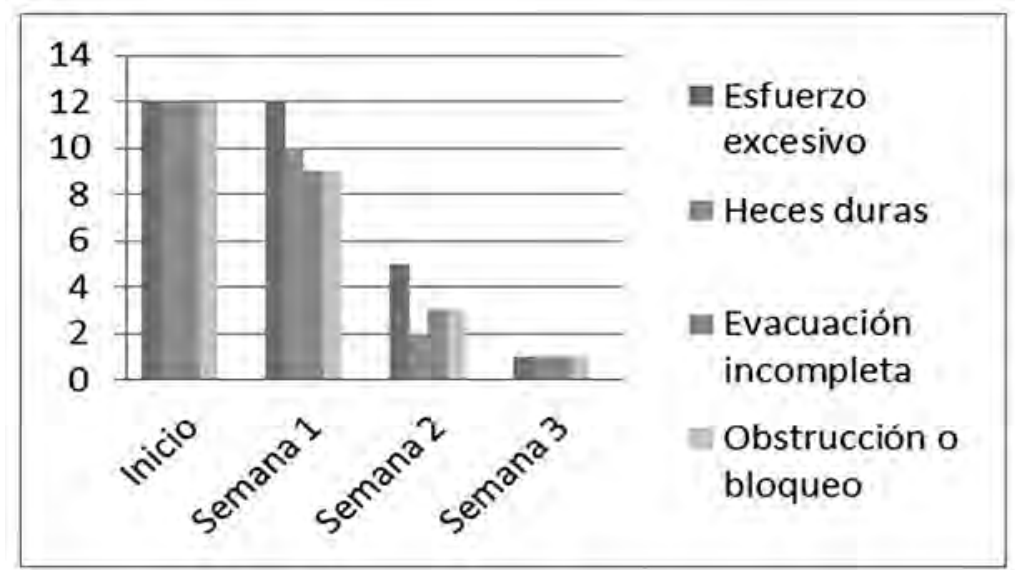

Figura 2

Síntomas de estreñimiento al inicio y las tres semanas de tratamiento del subgrupo A

En la figura 2 del subgrupo A se observa que al inicio del tratamiento todos presentaron los síntomas de estreñimiento, en la primera y segunda semana ya se observa una disminución de los síntomas de estreñimiento y en la tercera semana solo una persona prevalece con todos los síntomas de estreñimiento.

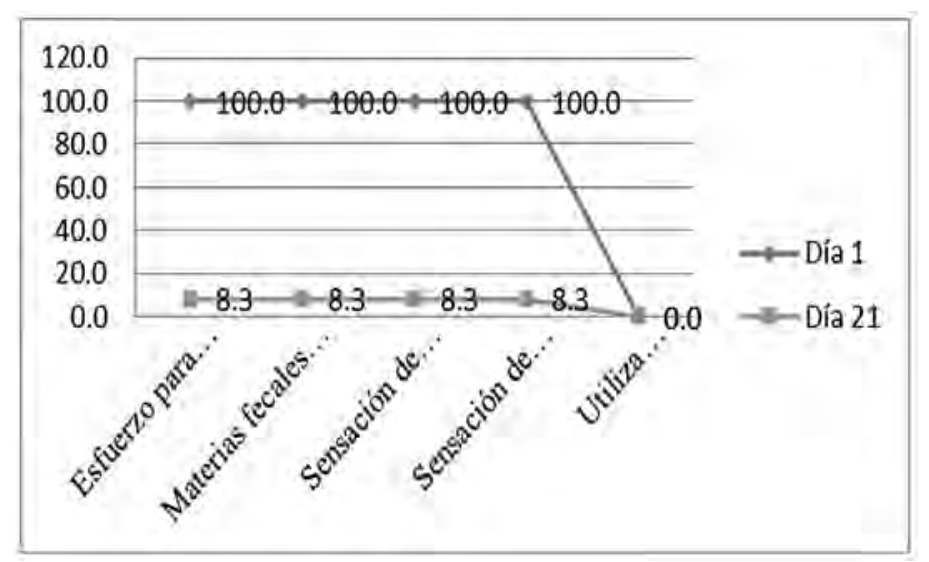

Figura 3

Comparación de los síntomas de estreñimiento antes y después del consumo de Chía del sub grupo A.

En el figura 3, se observa que de los 12 participantes que pertenecen al subgrupo $\mathrm{A}$, al inicio del tratamiento el $100 \%$ presentaron los síntomas de estreñimiento y al finalizar el tratamiento solo el $8.3 \%$ presentó todos los síntomas de estreñimiento, a excepción del síntoma de estreñimiento de utilización de maniobras manuales o digitales para defecar que no se presentó tanto al inicio y final del tratamiento. 
Efecto del consumo de chía (Salvia hispánica) sobre los síntomas de estreñimiento que presentan los estudiantes de una universidad particular de Lima Este, 2014

Tabla 1

Resultado de la prueba de McNemar del subgrupo A

\begin{tabular}{ll}
\hline Estadísticos de contraste McNemar & Inicio y final \\
\hline $\mathrm{N}$ & 12 \\
$\mathrm{p}$ & .001 \\
\hline
\end{tabular}

En la Tabla 1 se presenta la comparación entre el antes y después de la aplicación del tratamiento (Consumo de Chia), en la que se observa diferencias estadísticamente significativas $(\mathrm{p}<.05)$, lo cual permite señalar que el consumo de chía disminuyó los síntomas de estreñimiento del subgrupo A.

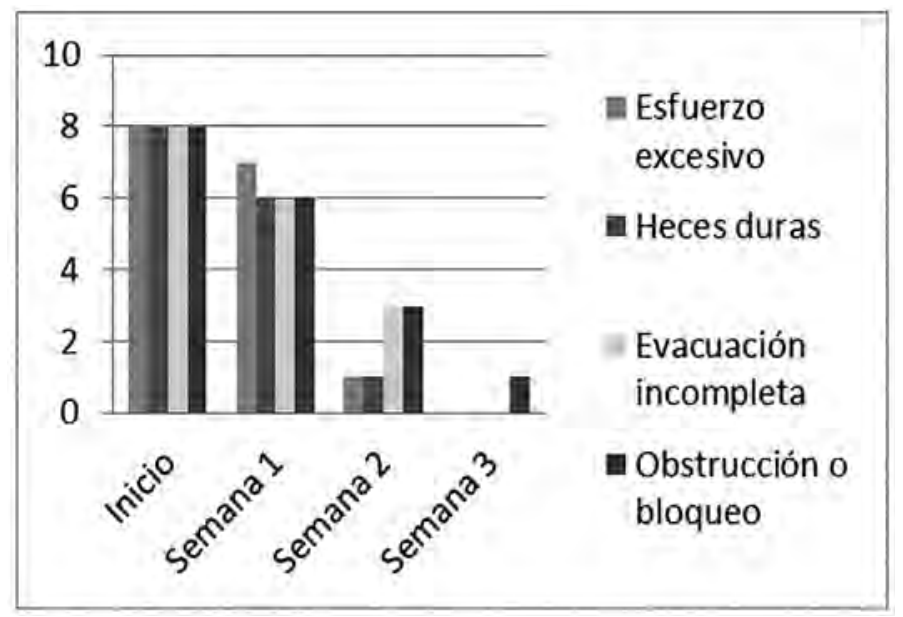

Figura 4

Síntomas de estreñimiento al inicio y las tres semanas de tratamiento del subgrupo B.

En la figura 4 del subgrupo B se observa que al inicio del tratamiento todos presentaron los síntomas de estreñimiento, en la primera y segunda semana ya se observa una disminución de los síntomas de estreñimiento y en la tercera semana solo una persona prevalece con un síntoma de estreñimiento (obstrucción o bloqueo).

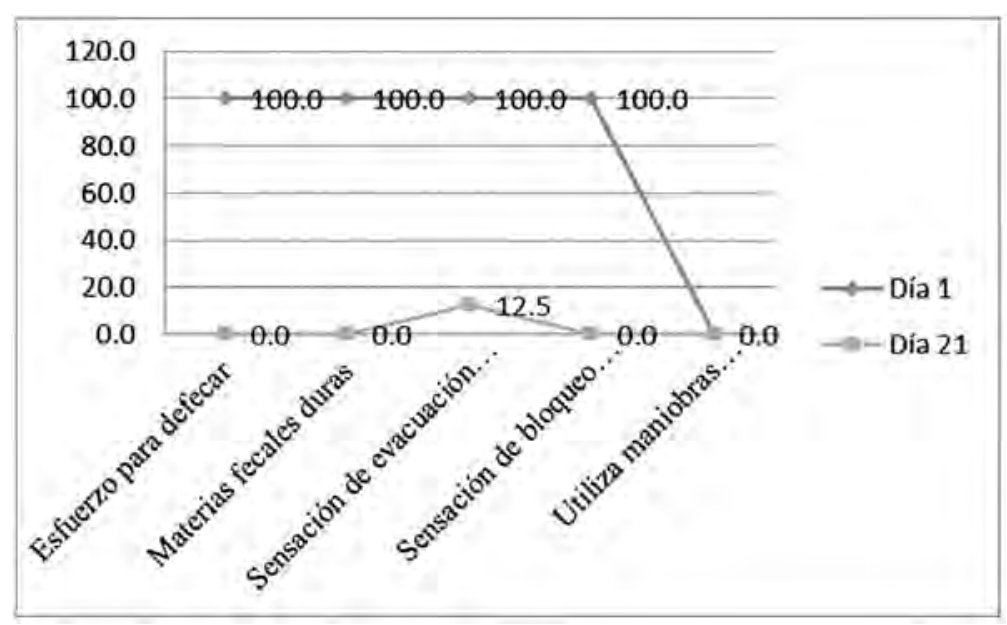

Figura 5

Comparación de los síntomas de estreñimiento antes y después del consumo de Chía del subgrupo.

Con respecto de la efectividad del consumo de chía en la disminución de los síntomas de estreñimiento en la presente figura se observa que de los 8 participantes que pertenecen al subgrupo $\mathrm{B}$, al inicio del tratamiento el $100 \%$ presentaron los síntomas de estreñimiento y al finalizar el tratamiento ninguno presento los síntomas de estreñimiento, a excepción del $12.5 \%$ que presentó solo el síntoma de estreñimiento de sensación de evacuación incompleta. 
Tabla 2

Resultado de la prueba de McNemar del subgrupo B.

\begin{tabular}{cc}
\hline Estadísticos de contraste McNemar & Basal y final \\
\hline $\mathrm{N}$ & 8 \\
$\mathrm{p}$-valor & .008 \\
\hline
\end{tabular}

En la Tabla 2 se presenta la comparación entre el antes y después de la aplicación del tratamiento (Consumo de Chía), en la que se observa diferencias estadísticamente significativas $(\mathrm{p}<.05)$, lo cual permite señalar que el consumo de chía disminuyó los síntomas de estreñimiento del subgrupo B.

\section{DISCUSIÓN}

La investigación tuvo como propósito determinar la efectividad del consumo de chía (Salvia hispánica) en la disminución de los síntomas de estreñimiento que presentaron los estudiantes universitarios, identificando los síntomas de estreñimiento antes y después del tratamiento del consumo de chía. Además, se identificaron las variables intervinientes (consumo de fibra dietética, actividad física y consumo de agua) copartícipe de la variable dependiente.

Según el diagrama de tendencia que se realizó, al inicio del tratamiento todos presentaron los síntomas de estreñimiento. Luego, en la primera y segunda semana del tratamiento ya se observa una disminución considerable de los síntomas de estreñimiento, y en la tercera semana solo dos personas de los subgrupos A y B presentaban los síntomas de estreñimiento. Además, se encontró que existe diferencia significativa entre el pre y pos test con respecto a los síntomas de estreñimiento de los participantes de este estudio, siendo corroborado con la prueba de McNemar $(\mathrm{p}<0.05)$.

Estos resultados son similares con un estudio realizado en México, cuyo objetivo fue disminuir los síntomas de estreñimiento en un grupo de adultos mayores mediante el consumo de tortilla de chía, concluyendo que hay disminución de los síntomas de estreñimiento con todas las dosis, principalmente con la dosis de $9 \mathrm{~g}$; sin embargo mediante el uso de la prueba estadística Chi Cuadrado, la disminución de síntomas fueron estadísticamente significativos a partir de la dosis de $6 \mathrm{~g}$ (dos tortilla s diarias) (Bautista et al. 2012).

De acuerdo a estos resultados se asume que la semilla de chía (Salvia hispánica) tiene efectos fisiológicos en el estreñimiento por su excelente fuente de fibra (27.6 gr de fibra en

100 gr de chía). La semilla de chía tiene un efecto beneficioso debido al equilibrado contenido de fibra: soluble (46.55\%) e insoluble $(53.45 \%)$ en comparación con otras fuentes de fibra como la de soja y la maíz, que de acuerdo al tipo de fibra presente poseen diferencias en la capacidad de fermentación y por lo tanto en los efectos fisiológicos que desarrollan, debido a que la fibra de la soja es altamente fermentable y digestible, al contrario de la fibra de maíz que es dura y difícil de degradar (Salgado et, al. 2005).

Entre las principales funciones fisiológicas de la chía (Salvia hispánica) podemos mencionar su capacidad para aumentar el bolo fecal y el metabolismo de los lípidos. Aumenta el volumen del bolo fecal, por su contenido en fibra dietética, absorbiendo grandes cantidades de agua, lo cual ayuda a prevenir problemas de estreñimiento, divertículos y cáncer de colón (Alvarado, 2011).

Este aumento se debe a la acción de su fibra soluble la cual se conforma de mucílagos, que tiene como característica principal la capacidad para atraer agua, porque exuda rápidamente al remojar las semillas en agua y se solubiliza fácilmente aún a bajas temperaturas formando geles, influyendo en el correcto funcionamiento intestinal (Alvarado, 2011 \& Garda, 2012).

Otras de las funciones fisiológicas de la ingesta de mucílago de chía es la influencia en el metabolismo de lípidos, mediante la disminución de la absorción intestinal de ácidos grasos, colesterol y el arrastre de sales biliares, aumentando la pérdida de colesterol a través de las heces, además inhibe la síntesis endógena de colesterol y la desaceleración de la digestión y la absorción de nutrientes (Guiotto, 2014).

Además, la fibra dietética soluble origina geles de alta viscosidad que producen enlentecimiento del vaciado gástrico y brinda sensación de saciedad (Guiotto, 2014).

Existen otras variables que intervienen en la 

versidad particular de Lima Este, 2014

disminución de los síntomas de estreñimiento, los cuales son el consumo de agua, la fibra dietaría y el ejercicio físico. Tal es el caso de los participantes del estudio, pues el 75\% consumen menos de 7 gr de fibra al día, este consumo estaría relacionado con los síntomas de estreñimiento de los participantes al inicio del estudio, ya que la fibra desempeña un papel primordial en el mantenimiento de la flora intestinal, la cantidad de bacterias y la excreción del bolo fecal (García, et al. 2002).

Así se evidencia en el estudio de López, et, alt. (2008), en España, quienes prepararon un lácteo con 20 gramos de fibra dietética (inulina y maltodextrina resistente a la digestión), mejorando la situación de estreñimiento crónico primario idiopático en base a los criterios de Roma III a un grupo de pacientes.

Así mismo, De los Ángeles (2011) reportó que en el colon es donde la fibra ejerce sus máximos efectos, además de diluir el contenido intestinal, sirve de substratos para la flora bacteriana, capta agua y fija cationes.

Otro de los factores que estarían interviniendo es el consumo de agua. Se observa que el65\% de los participantes refieren tomar de 1 a 2 litros de agua al día, lo cual facilitaría la eficacia del consumo de chía mejorando el transito gastrointestinal, como menciona Marien \& col (2012) que el consumo de fibra sin el aporte paralelo de suficiente líquido, empeora el curso y pronóstico del estreñimiento, la teoría científica demuestra que el consumo de agua como mínimo es de cuatro a seis vasos al día, siendo la ingesta dietética recomendada entre seis a ocho vasos de agua al día (Rubio, 2002).
Considerando la última variable interviniente, se encontró que el 55\% no realiza ejercicio físico y de los que lo practican el tiempo promedio es de 30 minutos en la semana. Al respecto, Vargas \& col (2013), mencionan que el tipo más frecuente de estreñimiento es el denominado estreñimiento crónico y esto se debe a la escasa actividad física, la monotonía en la dieta, y la inadecuada combinación de los alimentos, así como el consumo insuficiente de agua.

Al respecto, Paccor (2012) realizó un estudio sobre la frecuencia de la práctica de actividad física donde se identificó que el riesgo de padecer estreñimiento disminuye en un 35\% cuando se practica ejercicio de 2 a 6 veces por semana.

Se concluye que se encontró que existe diferencia significativa entre el pre y pos test con respecto a los síntomas de estreñimiento de los participantes de este estudio, siendo corroborado con la prueba de McNemar $(\mathrm{p}<0.05)$.

\section{Declaración de financiamiento y de conflicto de intereses:}

El estudio fue financiado por los autores, quienes declaran no tener algún tipo de conflicto de interés en la investigación realizada.

\section{Correspondencia:}

Johanna Jessenia Iñaguazo Trávez

Colegio Adventista Emanuel

Vía Quito Km. 2 - Barrio Napo - Lago Agrio, Quito, Ecuador. Tel. 593 (06) 2362497

e-mail: jessenia_johanna123@hotmail.com 


\section{REFERENCIAS BIBLIOGRÁFICAS}

Alvarado, R. (2011). Caracterización de la semilla de chan (Salvia hipanica L.) y diseño de un producto funcional que contiene como ingrediente. Revista 23 de la Universidad del Valle Guatemala., 43-49.

Bautista, M., Cervantes, E., Ramírez, A. y Sánchez Y. (2012). Disminución de síntomas de estreñimiento del adulto mayor mediante el consumo de tortilla de chía. Recuperado de Cyber Tesis: http://www. iberopuebla.edu.mx/micrositios/acisa/docsASE/ Otono2011/Estre\%C3\%Bimiento\%20tortilla\%20 de\%20ch\%c3\%ADa.pdf

Botella, R., Alfaro, M., Hernández, L., y Quílez T. (2011). Estrategias nutricionales ante el estreñimiento y la deshidratación en las personas mayores. Revista Nutrición Hospitalaria, 44-51.

De los Ángeles, B. (10 de Octubre de 2011). Formulación de una base para aderezo de ensaladas con características de alimento funcional. Recuperado de Cyber Tesis: http://posgrado.frba.utn.edu.ar/investigacion/tesis/ MTA-2011- Borda.pdf

Fuentes, A. (2012). Propiedades funcionales de la harina de semilla de chía (salvia hispanica l) y su efecto sobre las propiedades fisicoquímicas y microscópicas en un batido cárnico. Recuperado de Cyber Tesis: http://148.206.53.84/tesiuami/UAMI15754.pdf

García, F. (2012). Investigación Comercial. Tercera edición. Madrid, España.: Editorial ESIC.

García, P., Bretón, L., De la Cuerda, C. y Camblor, A. (2002). Metabolismo colónico de la fibra. Revista Nutrición Hospitalaria, 11-16.

García, P., Bretón, L., De la Cuerda, C. y Camblor, A. (2002). Metabolismo colónico de la fibra. Revista Nutrición Hospitalaria, 11-16.

Garda, M. A. (2012). Rol de los hidrocoloides de semillas de chía y lino en la optimización de panificados libres de gluten. Recuperado de Cyber Tesis: http://pesquisa. bvsalud.org/po

Gonzales, J. (2010). Caracterización de compuestos fenólicos presentes en la semilla y aceite de chía (salvia hispanica L.), mediante electroforesis capilar. Recuperado de Cyber Tesis: http://tesis.ipn.mx/jspui/ bitstream/123456789/9536/1/36.pdf

Guiotto, E. (2014). Aplicación de subproductos de chía (salvia hispanica l.) y girasol (helianthus annuus l.) en alimentos. [Tesis doctoral]. Argentina: Universidad de la Plata. Facultad de Ciencias Exactas. Recuperado de Cyber Tesis: http://sedici.unlp.edu.ar/bitstream/ handle/10915/34268/Documento_completo.pdf?seq uence $=3$

Higgins, P. J. (2004). Epidemiology of constipation in North America. Review Sysrematic, 1-26.

López, R., Martínez, G., Luque, J., Pons, M., Vargas, A., Iglesias, R., Hernández, M., Villegas, J. (2008). Efecto de la ingesta de un preparado lácteo con fibra dietética sobre el estreñimiento crónico primario idiopático. Rev. Nutrición hospitalaria, 12-19.

Lujan, O. (2011). Productos panaderos con agregados de harina de chía. Recuperado de Cyber Tesis: http://redi.ufasta.edu.ar:8080/xmlui/bitstream/ handle/123456789/340/2011_n_047.pdf ?sequence=1

Mearin, F., Balboa, A., Montoro, M. . (2012). Estreñimiento. Barcelona, España.: Instituto de trastornos funcionales y motores digestivos.

MINSA. (2004). Cifras sobre Estreñimiento. Recuperado de Ministerio de Salud del Perú: http://www.minsa.gob.pe

Paccor, A. (2012). Estado nutricional y hábitos alimentarios en estudiantes de cuarto año de la carrera de licenciatura en nutrición. Universidad Abierta Interamericana. Recuperado de Cyber Tesis: http://imgbiblio.vaneduc. edu.ar/fulltext/files/

Remes, T., Carmona, S., González, G., Martínez, S., Gómez, E., Ramírez, A., Vázquez, J., García, L., Abarca, C. y Roesch, D. (2009). ¿Qué se entiende por estreñimiento? Un estudio en población abierta. Revista Gastroenterología. México, 1-8.

Reyes-Caudillo, E., Tecante, A., Valdivia-López, M. (2008). Dietary fibre content and antioxidant activity of phenolic compounds present in Mexican chia (Salvia Hispanica L.) seeds, Food Chemistry. Clinical Nutrition, 656-663.

Rivera, B. (2006). Hábitos alimentarios en estudiantes de la Universidad Juárez Autónoma de Tabasco. Revista Cubana Salud Pública, 1-8.

Royo, V. (2007). Nutrición en Salud Pública. España: Instituto de Salud Carlos III. Rubio, M. (2002). Implicaciones de la fibra en distintas patologías. Revista Nutrición Hospitalaria, 17-29.

Salgado, C., Cedillo, L., Beltrán, O. (2005). Estudio de las Propiedades Funcionales de la Semilla de Chía (Salvia hispánica) y de la Fibra Dietaría Obtenida de la Misma. VII Congreso Nacional de Ciencia de los Alimentos y III Foro de Ciencia de los Alimentos (págs. 358-365). Nuevo León. México: Universidad Autónima de Nuevo León.

Tomas, M. (2013). Caracterización y funcionalidad de subproductos de chía (salvia hispanica L.) aplicación en tecnología de alimentos. [Tesis doctoral]. Recuperado de Cyber Tesis: http://sedici.unlp.edu.ar/bitstream/ handle/10915/26984/Documento_completo.\%20Ca pitani\%20(SP).pdf?sequence=1

Vargas, G. (2013). Consumo alimentario, estado nutricio y nivel de actividad física entre adultos mayores con y sin estreñimiento crónico. Revista Cirugía y Cirujanos, 214-220.

Recibido: 14/04/2015 Aceptado: 25/08/2015 